\title{
A GENERAL INVESTIGATION OF ADMISSIBLE COUPLINGS BETWEEN SYSTEMS OF HIGHER ORDER AND DIFFERENT TYPE
}

\author{
STEFAN EBENFELD \\ Darmstadt University of Technology \\ Department of Mathematics \\ Schlossgartenstr. 7, 64289 Darmstadt, Germany \\ E-mail: stefan.ebenfeld@d-fine.de
}

1. Introduction. In the paper at hand we study coupled systems of nonlinear initial boundary value problems where each system is of higher order, and of hyperbolic or parabolic type.

Our goal is to characterize systematically all admissible couplings between the single systems.

By an admissible coupling we mean a condition that guarantees the existence, uniqueness and regularity of solutions to the respective initial boundary value problem. For the proofs of our results we refer the reader to [3], [4] and [5].

The paper is organized as follows. In Section 1.1 we consider an example in order to illustrate the goal of our investigations. In Section 2 we formulate the initial boundary value problems under consideration. Moreover, we list some problems from continuum mechanics to which our general theory is applicable. In Section 3 we formulate the assumptions and the theorems about existence, uniqueness and regularity of solutions. In the above sense the regularity assumptions about the coefficients of the differential operators and about the right hand sides define the admissible couplings. In Section 4 we give a sketch of proof of our main theorem that involves the energy method. In Section 5 we specify the assumptions of our theory for the case of two coupled systems. Finally, in Section 6 we show some limitations of our theory.

2000 Mathematics Subject Classification: 35G20, 35M10.

The paper is in final form and no version of it will be published elsewhere. 
1.1. An example. As an example we consider the following initial boundary value problem:

$$
\begin{aligned}
\partial_{t}^{2} u(x, t)+\sum_{i=1}^{n} \partial_{i} F_{i}\left(\mathrm{D}_{x} u(x, t)\right. & \left., \mathrm{D}_{x}^{M_{v}^{F}} v(x, t), x, t\right) \\
& =f\left(\mathrm{D}_{x} u(x, t), \partial_{t} u(x, t), \mathrm{D}_{x}^{M_{v}^{f}} v(x, t), x, t\right)
\end{aligned}
$$

$$
\begin{aligned}
\partial_{t} v(x, t)+\sum_{i=1}^{n} \partial_{i} G_{i}\left(\mathrm{D}_{x}^{M_{u}^{G, 0}} u(x, t), \mathrm{D}_{x}^{M_{u}^{G, 1}} \partial_{t} u(x, t), \mathrm{D}_{x} v(x, t), x, t\right) \\
=g\left(\mathrm{D}_{x}^{M_{u}^{g, 0}} u(x, t), \mathrm{D}_{x}^{M_{u}^{g, 1}} \partial_{t} u(x, t), \mathrm{D}_{x} v(x, t), x, t\right) .
\end{aligned}
$$

$(\mathrm{BC})$

$$
\left.u(x, t)\right|_{x \in \partial \Omega}=0,\left.\quad v(x, t)\right|_{x \in \partial \Omega}=0 .
$$

$$
u(x, 0)=0, \quad \partial_{t} u(x, 0)=0, \quad v(x, 0)=0 .
$$

In the above example $(\mathrm{H})$ is a hyperbolic PDE of order two for $u$ whereas $(\mathrm{P})$ is a parabolic PDE of order two for $v$. The parameters $M$ describe the strength of the coupling between the two systems. We note that throughout this paper we will restrict our attention to zero Dirichlet boundary conditions and zero initial conditions. For the above example the question of the admissibility of the coupling reads as follows:

Let the constitutive functions $F_{i}, f, G_{i}$ and $g$ satisfy suitable structural conditions (such as smoothness, ellipticity and compatibility). For which values of the parameters $M$ does the above initial boundary value problem have a unique smooth local in time solution $(u, v)$ ?

Specifying our general theory to the above example we obtain the following answer:

The above initial boundary value problem has a unique smooth local in time solution $(u, v)$ for the following choices of the parameters $M$ :

$$
\begin{aligned}
& M_{v}^{F} \leq 0, \quad M_{v}^{f} \leq 1, \quad M_{u}^{G, 0} \leq 1, \quad M_{u}^{G, 1} \leq 0, \\
& M_{u}^{g, 0} \leq 2, \quad M_{u}^{g, 1} \leq 1
\end{aligned}
$$

or

$$
\begin{aligned}
& M_{v}^{F} \leq 1, \quad M_{v}^{f} \leq 2, \quad M_{u}^{G, 0} \leq 0, \quad M_{u}^{G, 1}=-\infty, \\
& M_{u}^{g, 0} \leq 1, \quad M_{u}^{g, 1} \leq 0 .
\end{aligned}
$$

In the above inequalities the notation $M_{u}^{G, 1}=-\infty$ means that the constitutive functions $G_{i}$ are independent of $\partial_{t} u$. We note that the first choice of the parameters $M$ covers the case of nonlinear thermoelasticity, cf. [6] (Jiang-Racke).

2. Statement of the problem. Let $T>0$, and let $\Omega \subset \mathbb{R}^{n}$ be a bounded domain with a smooth boundary. We consider the following functions:

$$
u_{j i_{j}}: \overline{\Omega \times(0, T)} \longrightarrow \mathbb{R}^{N_{j i_{j}}}:(x, t) \longmapsto u_{j i_{j}}(x, t) \quad\left(j=1,2,3, i_{j}=1, \ldots, I_{j}\right) .
$$


Throughout this paper we will use a double index notation to enumerate the systems under consideration. The first index $j$ indicates the type of the system:

$j=1 \quad$ indicates a hyperbolic system satisfying a symmetry condition.

$j=2 \quad$ indicates a parabolic system satisfying no symmetry condition.

$j=3 \quad$ indicates a parabolic system satisfying a symmetry condition.

The second index $i_{j}$ enumerates the systems of type $j$.

2.1. The abstract problem. We make the following definition:

$$
\boldsymbol{A}_{j i_{j}}[u](\nabla, x, t) u_{j i_{j}}:=\sum_{|\alpha|,|\beta|=0}^{m_{j i_{j}}}(-1)^{|\alpha|} \partial_{x}^{\alpha}\left(A_{j i_{j}, \alpha \beta}[u](x, t) \partial_{x}^{\beta} u_{j i_{j}}\right) .
$$

In the above definition the square bracket notation $\Phi[u]$ means that $\Phi$ acts as a nonlinear operator on the functions $u_{j i_{j}}$. In particular, $\boldsymbol{A}_{j i_{j}}[u](\nabla, x, t)$ denotes a differential operator of order $2 m_{j i_{j}}$ with coefficients depending on $u_{11}, \ldots, u_{3 I_{3}}$. We consider the following abstract initial boundary value problem:

$$
\begin{aligned}
& \partial_{t}^{2} u_{1 i_{1}}+\boldsymbol{A}_{1 i_{1}}[u](\nabla, x, t) u_{1 i_{1}}=f_{1 i_{1}}[u](x, t) . \\
& \partial_{t} u_{2 i_{2}}+\boldsymbol{A}_{2 i_{2}}[u](\nabla, x, t) u_{2 i_{2}}=f_{2 i_{2}}[u](x, t) . \\
& \partial_{t} u_{3 i_{3}}+\boldsymbol{A}_{3 i_{3}}[u](\nabla, x, t) u_{3 i_{3}}=f_{3 i_{3}}[u](x, t) . \\
& \left.\partial_{x}^{\alpha} u_{j i_{j}}\right|_{x \in \partial \Omega}=0 \quad\left(|\alpha|=0, \ldots, m_{j i_{j}}-1\right) . \\
& \left.u_{1 i_{1}}\right|_{t=0}=0,\left.\quad \partial_{t} u_{1 i_{1}}\right|_{t=0}=0,\left.\quad u_{2 i_{2}}\right|_{t=0}=0,\left.\quad u_{3 i_{3}}\right|_{t=0}=0 .
\end{aligned}
$$

According to our notation $(\mathrm{H})$ is a collection of hyperbolic PDEs for the functions $u_{1 i_{1}}$ whereas (P1) and (P2) are collections of parabolic PDEs for the functions $u_{2 i_{2}}$ and $u_{3 i_{3}}$ respectively.

2.2. The hyperbolic-parabolic problem. As an application we consider the following hyperbolic-parabolic initial boundary value problem that generalizes the example given in the introduction:

$$
\begin{aligned}
& \partial_{t}^{2} u_{1 i_{1}}+\sum_{|\alpha|=0}^{m_{1 i_{1}}}(-1)^{|\alpha|} \partial_{x}^{\alpha}\left(F_{1 i_{1}, \alpha}\left(U_{1 i_{1}}^{F}, x, t\right)\right)=f_{1 i_{1}}\left(U_{1 i_{1}}^{f}, x, t\right) . \\
& \partial_{t} u_{2 i_{2}}+\sum_{|\alpha|=0}^{m_{2 i_{2}}}(-1)^{|\alpha|} \partial_{x}^{\alpha}\left(F_{2 i_{2}, \alpha}\left(U_{2 i_{2}}^{F}, x, t\right)\right)=f_{2 i_{2}}\left(U_{2 i_{2}}^{f}, x, t\right) \text {. } \\
& \partial_{t} u_{3 i_{3}}+\sum_{|\alpha|=0}^{m_{3 i_{3}}}(-1)^{|\alpha|} \partial_{x}^{\alpha}\left(F_{3 i_{3}, \alpha}\left(U_{3 i_{3}}^{F}, x, t\right)\right)=f_{3 i_{3}}\left(U_{3 i_{3}}^{f}, x, t\right) . \\
& \left.\partial_{x}^{\alpha} u_{j i_{j}}\right|_{x \in \partial \Omega}=0 \quad\left(|\alpha|=0, \ldots, m_{j i_{j}}-1\right) . \\
& \left.u_{1 i_{1}}\right|_{t=0}=0,\left.\quad \partial_{t} u_{1 i_{1}}\right|_{t=0}=0,\left.\quad u_{2 i_{2}}\right|_{t=0}=0,\left.\quad u_{3 i_{3}}\right|_{t=0}=0 .
\end{aligned}
$$


In the above initial boundary value problem the $U_{j i_{j}}^{\phi}$ denote the collections of partial derivatives of the functions $u_{11}, \ldots, u_{3 I_{3}}$ that occur in the constitutive functions $\phi_{j i_{j}}$ where $\phi=F, f$.

2.3. Applications in continuum mechanics. The single PDE systems in our initial boundary value problems are suitable to describe a wide range of problems in continuum mechanics. Some of them are the following:

Elasticity (hyperbolic, second order).

Viscoelasticity (hyperbolic, second order).

Higher gradient materials (hyperbolic, higher order).

Shell theory-Reissner Mindlin Type (hyperbolic, second order).

Shell theory-Kirchhoff Love Type (hyperbolic, higher order).

Heat flow (parabolic, second order).

Heat flow-Müller Type (hyperbolic, second order).

Compressible fluid flow (parabolic, second order).

Multipolar compressible fluids (parabolic, higher order).

Magnetodynamics (parabolic, second order).

Phase transitions - Cahn Allen Type (parabolic, second order).

Phase transitions - Cahn Hilliard Type (parabolic, higher order).

We note that the mathematical structure of some of the above problems can be identical, but their coupling with other problems can be quite different. Moreover, we note that many of the above problems immediately fit into the scheme of our hyperbolicparabolic problem. However, some other problems have to be transformed and fit only into the scheme of our abstract problem, such as viscoelasticity and compressible fluid flow.

\section{Statement of the theorem}

3.1. The abstract problem. For the case of the abstract initial boundary value problem problem we make the following assumptions:

(A1) Symmetry condition $(j=1,3)$ :

$$
A_{j i_{j}, \beta \alpha}[u](x, t)=\left(A_{j i_{j}, \alpha \beta}[u](x, t)\right)^{T} .
$$

(A2) Legendre-Hadamard condition (strong ellipticity):

$$
\sum_{|\alpha|,|\beta|=m_{j i_{j}}} \eta^{T}\left(A_{j i_{j}, \alpha \beta}[u](x, t) \xi^{\alpha} \xi^{\beta}\right) \eta \geq c|\xi|^{2 m_{j i_{j}}}|\eta|^{2} .
$$

(A3) Compatibility condition:

$$
\begin{array}{rrr}
\left.\partial_{t}^{\kappa} u_{1 i_{1}}\right|_{t=0}=0, & \left.\partial_{t}^{\kappa+1} u_{1 i_{1}}\right|_{t=0}=0, \\
\left.\partial_{t}^{\kappa} u_{2 i_{2}}\right|_{t=0}=0, & \left.\partial_{t}^{\kappa} u_{3 i_{3}}\right|_{t=0}=0 & (\kappa=0, \ldots, k+1) \\
& \left.\Longrightarrow \partial_{t}^{k} f_{j i_{j}}[u]\right|_{t=0}=0 .
\end{array}
$$


(A4) Boundedness condition:

$$
\left\|u_{l k_{l}}\right\|_{\mathcal{U}_{j i_{j}, l k_{l}}\left(T^{*}\right)} \leq R \Longrightarrow \begin{aligned}
& \left\|A_{j i_{j}, \alpha \beta}[u]\right\|_{\mathcal{A}_{j i_{j}}\left(T^{*}\right)} \leq \Phi\left(R, T^{*}\right) \\
& \left\|f_{j i_{j}}[u]\right\|_{\mathcal{F}_{j i_{j}}\left(T^{*}\right)} \leq \Phi\left(R, T^{*}\right) .
\end{aligned}
$$

(A5) Lipschitz condition:

$$
\begin{aligned}
& \left\|u_{l k_{l}}^{1}\right\|_{\overline{\mathcal{U}}_{j i_{j}, l k_{l}}\left(T^{*}\right)},\left\|u_{l k_{l}}^{2}\right\|_{\overline{\mathcal{U}}_{j i_{j}, l k_{l}}\left(T^{*}\right)} \leq R \\
& \left\|u_{l k_{l}}^{2}-u_{l k_{l}}^{1}\right\|_{\underline{\mathcal{U}}_{j i_{j}, l k_{l}}\left(T^{*}\right)} \leq S \\
& \quad\left\|A_{j i_{j}, \alpha \beta}\left[u^{2}\right]-A_{j i_{j}, \alpha \beta}\left[u^{1}\right]\right\|_{\mathcal{A}_{j i_{j}}\left(T^{*}\right)} \leq \Phi\left(R, T^{*}\right) S \\
& \Longrightarrow\left\|f_{j i_{j}}\left[u^{2}\right]-f_{j i_{j}}\left[u^{1}\right]\right\|_{\mathcal{F}_{j i_{j}}\left(T^{*}\right)} \leq \Phi\left(R, T^{*}\right) S .
\end{aligned}
$$

In the above assumptions the $\mathcal{U}_{j i_{j}, l k_{l}}\left(T^{*}\right), \overline{\mathcal{U}}_{j i_{j}, l k_{l}}\left(T^{*}\right), \underline{\mathcal{U}}_{j i_{j}, l k_{l}}\left(T^{*}\right), \mathcal{A}_{j i_{j}}\left(T^{*}\right)$ and $\mathcal{F}_{j i_{j}}\left(T^{*}\right)$ denote suitable function spaces. The assumptions (A1), (A2) and (A3) are structural conditions. The regularity assumptions (A4) and (A5) define the admissible couplings.

ThEOREM (Local Existence, Uniqueness, Regularity). Let the assumptions (A1)-(A5) be satisfied. Then there exists $0<T^{*} \leq T$ such that the abstract initial boundary value problem has a unique smooth solution:

$$
\begin{aligned}
& u_{1 i_{1}} \in \bigcap_{k=0}^{\bar{k}-1} \mathcal{C}^{\bar{l}+k}\left(\left[0, T^{*}\right], H^{\mu(\bar{k}-1-k)+2 m_{1 i_{1}}}\left(\Omega, \mathbb{R}^{N_{1 i_{1}}}\right)\right) \\
& \cap \mathcal{C}^{\bar{l}+\bar{k}}\left(\left[0, T^{*}\right], H_{0}^{m_{1 i_{1}}}\left(\Omega, \mathbb{R}^{N_{1 i_{1}}}\right)\right) \cap \mathcal{C}^{\bar{l}+\bar{k}+1}\left(\left[0, T^{*}\right], L^{2}\left(\Omega, \mathbb{R}^{N_{1 i_{1}}}\right)\right) . \\
& u_{2 i_{2}} \in \bigcap_{k=0}^{\bar{k}-1} \mathcal{C}^{\bar{l}+k}\left(\left[0, T^{*}\right], H^{\mu(\bar{k}-1-k)+m_{2 i_{2}}+m_{2 i_{2} 0}}\left(\Omega, \mathbb{R}^{N_{2 i_{2}}}\right)\right) \\
& \cap \mathcal{C}^{\bar{l}+\bar{k}}\left(\left[0, T^{*}\right], L^{2}\left(\Omega, \mathbb{R}^{N_{2 i_{2}}}\right)\right) \cap H^{\bar{l}+\bar{k}}\left(\left[0, T^{*}\right], H_{0}^{m_{2 i_{2}}}\left(\Omega, \mathbb{R}^{N_{2 i_{2}}}\right)\right) \\
& \cap H^{\bar{l}+\bar{k}+1}\left(\left[0, T^{*}\right], H^{-m_{2 i_{2}}}\left(\Omega, \mathbb{R}^{N_{2 i_{2}}}\right)\right) \text {. } \\
& u_{3 i_{3}} \in \bigcap_{k=0}^{\bar{k}-1} \mathcal{C}^{\bar{l}+k}\left(\left[0, T^{*}\right], H^{\mu(\bar{k}-1-k)+m_{3 i_{3}}+m_{3 i_{3} 0}}\left(\Omega, \mathbb{R}^{N_{3 i_{3}}}\right)\right) \\
& \cap \mathcal{C}^{\bar{l}+\bar{k}}\left(\left[0, T^{*}\right], H_{0}^{m_{3 i_{3}}}\left(\Omega, \mathbb{R}^{N_{3 i_{3}}}\right)\right) \cap H^{\bar{l}+\bar{k}}\left(\left[0, T^{*}\right], H^{2 m_{3 i_{3}}}\left(\Omega, \mathbb{R}^{N_{3 i_{3}}}\right)\right) \\
& \cap H^{\bar{l}+\bar{k}+1}\left(\left[0, T^{*}\right], L^{2}\left(\Omega, \mathbb{R}^{N_{3 i}}\right)\right) .
\end{aligned}
$$

We note that the scales of function spaces in the above theorem enter into our nonlinear theory in a natural way through the underlying linear theory.

3.2. The hyperbolic-parabolic problem. For the case of the hyperbolic-parabolic initial boundary value problem we make the following definition:

$$
A_{j i_{j}, \alpha \beta}[u](x, t):=\frac{\partial F_{j i_{j}, \alpha}}{\partial\left(\partial_{x}^{\beta} u_{j i_{j}}\right)}\left(U_{j i_{j}}^{F}, x, t\right) .
$$

Moreover, we use the following notation: 
$M_{j i_{j}, l k_{l}}^{\phi, 0}$ denotes the highest order of spatial derivatives of $u_{l k_{l}}$ that occurs in the constitutive functions $\phi_{j i_{j}}$ where $\phi=F, f$.

$M_{j i_{j}, 1 k_{1}}^{\phi, 1}$ denotes the highest order of spatial derivatives of $\partial_{t} u_{1 k_{1}}$ that occurs in the constitutive functions $\phi_{j i_{j}}$ where $\phi=F, f$.

We also make the following assumptions:

(B1) $F_{j i_{j}, \alpha}$ and $f_{j i_{j}}$ are smooth functions.

(B2) Symmetry condition $(j=1,3)$ :

$$
A_{j i_{j}, \beta \alpha}[u](x, t)=\left(A_{j i_{j}, \alpha \beta}[u](x, t)\right)^{T} .
$$

(B3) Legendre-Hadamard condition (strong ellipticity):

$$
\sum_{|\alpha|,|\beta|=m_{j i_{j}}} \eta^{T}\left(A_{j i_{j}, \alpha \beta}[u](x, t) \xi^{\alpha} \xi^{\beta}\right) \eta \geq c|\xi|^{2 m_{j i_{j}}}|\eta|^{2} .
$$

(B4) Compatibility condition:

$$
\left.\partial_{t}^{k}\left(f_{j i_{j}}(0, x, t)\right)\right|_{t=0}=0 \quad(k=0, \ldots, \bar{l}+\bar{k}) .
$$

(B5) Regularity assumptions (I):

$$
\begin{array}{lll}
M_{1 i_{1}, 1 i_{1}}^{F, 0}=m_{1 i_{1}}, & M_{1 i_{1}, 1 i_{1}}^{F, 1}=-\infty, & M_{1 i_{1}, 1 i_{1}}^{f, 0} \leq m_{1 i_{1}}, \\
M_{1 i_{1}, 1 i_{1}}^{f, 1} \leq 0 . & M_{2 i_{2}, 2 i_{2}}^{F, 0}=m_{2 i_{2}}, & M_{2 i_{2}, 2 i_{2}}^{f, 0} \leq 2 m_{2 i_{2}}-1 . \\
M_{3 i_{3}, 3 i_{3}}^{F, 0}=m_{3 i_{3}}, & M_{3 i_{3}, 3 i_{3}}^{f, 0} \leq 2 m_{3 i_{3}}-1 . &
\end{array}
$$

Regularity assumptions (II):

$$
M_{j i_{j}, l k_{l}}^{\phi, \nu} \leq \Phi_{j i_{j}, l k_{l}}^{\phi, \nu}\left(m_{j i_{j}} m_{l k_{l}}\right) .
$$

In the above assumptions the $\Phi_{j i_{j}, l k_{l}}^{\phi, \nu}$ denote suitable functions specified in [5]. The assumptions (B1), (B2), (B3) and (B4) are structural conditions. The regularity assumptions (B5) define the admissible couplings now. In particular, the regularity assumptions (B5) imply the previous regularity assumptions (A4) and (A5). We note that by Poincaré's lemma the symmetry condition (B2) is equivalent to the following integrability condition:

$$
F_{j i_{j}, \alpha}\left(U_{j i_{j}}^{F}, x, t\right)=\frac{\partial \Psi_{j i_{j}}}{\partial\left(\partial_{x}^{\alpha} u_{j i_{j}}\right)}\left(U_{j i_{j}}^{F}, x, t\right) .
$$

Corollary (Local Existence, Uniqueness, Regularity). Let (B1)-(B5) be satisfied. Then there exists $0<T^{*} \leq T$ such that the hyperbolic-parabolic initial boundary value problem has a unique smooth solution:

$$
\begin{aligned}
u_{1 i_{1}} \in \bigcap_{k=0}^{\bar{k}-1} \mathcal{C}^{\bar{l}+k+1}\left(\left[0, T^{*}\right], H^{\mu(\bar{k}-1-k)+2 m_{1 i_{1}}}\left(\Omega, \mathbb{R}^{N_{1 i_{1}}}\right)\right) \\
\quad \cap \mathcal{C}^{\bar{l}+\bar{k}+1}\left(\left[0, T^{*}\right], H_{0}^{m_{1 i_{1}}}\left(\Omega, \mathbb{R}^{N_{1 i_{1}}}\right)\right) \cap \mathcal{C}^{\bar{l}+\bar{k}+2}\left(\left[0, T^{*}\right], L^{2}\left(\Omega, \mathbb{R}^{N_{1 i_{1}}}\right)\right) .
\end{aligned}
$$




$$
\begin{aligned}
u_{2 i_{2}} \in \bigcap_{k=0}^{\bar{k}-1} \mathcal{C}^{\bar{l}+k+1}\left(\left[0, T^{*}\right], H^{\mu(\bar{k}-1-k)+m_{2 i_{2}}+m_{2 i_{2} 0}}\left(\Omega, \mathbb{R}^{N_{2 i_{2}}}\right)\right) \\
\\
\quad \cap \mathcal{C}^{\bar{l}+\bar{k}+1}\left(\left[0, T^{*}\right], L^{2}\left(\Omega, \mathbb{R}^{N_{2 i_{2}}}\right)\right) \cap H^{\bar{l}+\bar{k}+1}\left(\left[0, T^{*}\right], H_{0}^{m_{2 i_{2}}}\left(\Omega, \mathbb{R}^{N_{2 i_{2}}}\right)\right) \\
\quad \cap H^{\bar{l}+\bar{k}+2}\left(\left[0, T^{*}\right], H^{-m_{2 i_{2}}}\left(\Omega, \mathbb{R}^{N_{2 i_{2}}}\right)\right) . \\
u_{3 i_{3}} \in \bigcap_{k=0}^{\bar{k}-1} \mathcal{C}^{\bar{l}+k+1}\left(\left[0, T^{*}\right], H^{\left.\mu(\bar{k}-1-k)+m_{3 i_{3}}+m_{3 i_{3} 0}\left(\Omega, \mathbb{R}^{N_{3 i_{3}}}\right)\right)}\right. \\
\quad \cap \mathcal{C}^{\bar{l}+\bar{k}+1}\left(\left[0, T^{*}\right], H_{0}^{m_{3 i_{3}}}\left(\Omega, \mathbb{R}^{N_{3 i_{3}}}\right)\right) \cap H^{\bar{l}+\bar{k}+1}\left(\left[0, T^{*}\right], H^{2 m_{3 i_{3}}}\left(\Omega, \mathbb{R}^{N_{3 i_{3}}}\right)\right) \\
\cap H^{\bar{l}+\bar{k}+2}\left(\left[0, T^{*}\right], L^{2}\left(\Omega, \mathbb{R}^{N_{3 i_{3}}}\right)\right) .
\end{aligned}
$$

4. Sketch of proof (energy method). In order to prove our main theorem we make use of the so called energy method, see e.g. [1] (Dafermos-Hrusa), [7] (Kato) and [8] (Majda). Therefore, we proceed in several steps:

1. First, we define of a sequence $\left\{u^{\nu}\right\}_{\nu=0}^{\infty}$ of approximate solutions with the help of the following recursion scheme:

(a) Let $u^{0}:=0$.

(b) Insert the previous solution $u^{\nu}$ into the coefficients $A_{j i_{j}, \alpha \beta}[\cdot]$ and right hand sides $f_{j i_{j}}[\cdot]$.

(c) Solve the linearized initial boundary value problem for $u^{\nu+1}$.

The crucial point in this step is that we cannot make use of the full regularity of $u^{\nu}$ as we insert it into the $A_{j i_{j}, \alpha \beta}[\cdot]$ and $f_{j i_{j}}[\cdot]$. Therefore, we linearize the single systems successively in the following way, cf. [6] (Jiang-Racke):

$$
\begin{aligned}
& \partial_{t} v_{k}+\boldsymbol{B}_{k}[v](\nabla, x, t) v_{k}=g_{k}[v](x, t) \quad(k=1, \ldots, K) \\
& \Longrightarrow \quad \partial_{t} v_{k}^{\nu+1}+\boldsymbol{B}_{k}\left[\ldots, v_{k-1}^{\nu+1}, v_{k}^{\nu}, \ldots\right](\nabla, x, t) v_{k}^{\nu+1}=g_{k}\left[\ldots, v_{k-1}^{\nu+1}, v_{k}^{\nu}, \ldots\right](x, t) .
\end{aligned}
$$

2. Next, we derive a priori estimates for $\left\{u^{\nu}\right\}_{\nu=0}^{\infty}$ using Galerkin's method. In view of the regularity of the $u_{j i_{j}}^{\nu}$ we have the following correspondence:

$$
\begin{array}{llll}
u_{1 i_{1}}^{\nu} \longleftrightarrow & \text { testing } & \partial_{t}^{2} u+(-\Delta)^{m} u=f & \text { with } \partial_{t} u . \\
u_{2 i_{2}}^{\nu} \longleftrightarrow & \text { testing } & \partial_{t} u+(-\Delta)^{m} u=f & \text { with } u . \\
u_{3 i_{3}}^{\nu} \longleftrightarrow & \text { testing } & \partial_{t} u+(-\Delta)^{m} u=f & \text { with } \partial_{t} u .
\end{array}
$$

One crucial point in this step is that we need an elliptic regularity theory for linear elliptic differential operators with minimal regularity in the coefficients. Such a theory was previously developed by the author, see [2]. Another crucial point in this step is that we have to find suitable scales of function spaces. The important observation is that we can find some $\mu \geq 1$ and corresponding scales of function spaces such that for any $\left(j, i_{j}\right)$ one temporal derivative of $u_{j i_{j}}$ corresponds to $\mu$ spatial derivatives of $u_{j i_{j}}$, cf. [6] (Jiang-Racke).

3. Next, with the help of the above a priori estimates we show that the sequence $\left\{u^{\nu}\right\}_{\nu=0}^{\infty}$ is bounded with respect to some high norm and contractive with respect to 
some low norm.

4. Finally, we pass to the limit $\nu \longrightarrow \infty$. In particular, we show that the sequence $\left\{u^{\nu}\right\}_{\nu=0}^{\infty}$ converges to some limit function $u$ and that $u$ is the unique solution to the abstract initial boundary value problem. The crucial point in this step is that we make use of interpolation inequalities in non-dual spaces. Consequently, we cannot make direct use of Banach's Fixed Point Theorem (Kato's direct method) but we rather pass to the limit 'by hand', cf. [8] (Majda) and compare with [1] (Dafermos-Hrusa) and [7] (Kato).

5. Application to two coupled systems. We consider the hyperbolic-parabolic initial boundary value problem for the case of two coupled systems.

Definition. We say that the coupling in the hyperbolic-parabolic initial boundary value problem is admissible if the regularity assumptions (B5) hold.

We note that if the constitutive functions satisfy the structural conditions (B1)-(B4) and if the coupling in the hyperbolic-parabolic initial boundary value problem is admissible then by the above corollary the hyperbolic-parabolic initial boundary value problem has a unique smooth solution.

5.1. Hyperbolic-hyperbolic systems. We consider the initial boundary value problem corresponding to the following PDE systems:

$$
\begin{array}{r}
\partial_{t}^{2} u+\sum_{|\alpha|=0}^{m_{u}}(-1)^{|\alpha|} \partial_{x}^{\alpha} F_{\alpha}\left(\mathrm{D}_{x}^{m_{u}} u, \mathrm{D}_{x}^{M_{v}^{F, 0}} v, \mathrm{D}_{x}^{M_{v}^{F, 1}} \partial_{t} v, x, t\right) \\
=f\left(\mathrm{D}_{x}^{m_{u}} u, \partial_{t} u, \mathrm{D}_{x}^{M_{v}^{f, 0}} v, \mathrm{D}_{x}^{M_{v}^{f, 1}} \partial_{t} v, x, t\right) . \\
\begin{aligned}
\partial_{t}^{2} v+\sum_{|\alpha|=0}^{m_{v}}(-1)^{|\alpha|} \partial_{x}^{\alpha} G_{\alpha}\left(\mathrm{D}_{x}^{M_{u}^{G, 0}} u, \mathrm{D}_{x}^{M_{u}^{G, 1}} \partial_{t} u, \mathrm{D}_{x}^{m_{v}} v, x, t\right) \\
=g\left(\mathrm{D}_{x}^{M_{u}^{g, 0}} u, \mathrm{D}_{x}^{M_{u}^{g, 1}} \partial_{t} u, \mathrm{D}_{x}^{m_{v}} v, \partial_{t} v, x, t\right) .
\end{aligned}
\end{array}
$$

LEMMA. The coupling in the above initial boundary value problem is admissible for the following choice of the parameters $M$ :

$$
\begin{aligned}
& M_{v}^{F, 0} \leq m_{v}-m_{u}, \quad M_{v}^{F, 1}=-\infty, \quad M_{v}^{f, 0} \leq m_{v}, \quad M_{v}^{f, 1} \leq 0, \\
& M_{u}^{G, 0} \leq m_{u}-m_{v}, \quad M_{u}^{G, 1}=-\infty, \quad M_{u}^{g, 0} \leq m_{u}, \quad M_{u}^{g, 1} \leq 0 .
\end{aligned}
$$

5.2. Hyperbolic-parabolic systems. We consider the initial boundary value problem corresponding to the following PDE systems:

$$
\begin{aligned}
& \partial_{t}^{2} u+\sum_{|\alpha|=0}^{m_{u}}(-1)^{|\alpha|} \partial_{x}^{\alpha} F_{\alpha}\left(\mathrm{D}_{x}^{m_{u}} u, \mathrm{D}_{x}^{M_{v}^{F}} v, x, t\right)=f\left(\mathrm{D}_{x}^{m_{u}} u, \partial_{t} u, \mathrm{D}_{x}^{M_{v}^{f}} v, x, t\right) . \\
& \begin{aligned}
\partial_{t} v+\sum_{|\alpha|=0}^{m_{v}}(-1)^{|\alpha|} \partial_{x}^{\alpha} G_{\alpha}\left(\mathrm{D}_{x}^{M_{u}^{G, 0}} u, \mathrm{D}_{x}^{M_{u}^{G, 1}} \partial_{t} u, \mathrm{D}_{x}^{m_{v}} v, x, t\right) \\
= \\
=g\left(\mathrm{D}_{x}^{M_{u}^{g, 0}} u, \mathrm{D}_{x}^{M_{u}^{g, 1}} \partial_{t} u, \mathrm{D}_{x}^{2 m_{v}-1} v, x, t\right) .
\end{aligned}
\end{aligned}
$$


LEMMA. The coupling in the above initial boundary value problem is admissible for the following choices of the parameters $M$ :

$$
\begin{array}{ll}
M_{v}^{F} \leq m_{v}-m_{u}, & M_{v}^{f} \leq m_{v}, \\
M_{u}^{g, 0} \leq m_{u}+m_{v}, & M_{u}^{g, 1} \leq m_{v}
\end{array} \quad M_{u}^{G, 0} \leq m_{u}, \quad M_{u}^{G, 1} \leq 0,
$$

or

$$
\begin{array}{ll}
M_{v}^{F} \leq 2 m_{v}-m_{u}, \quad & M_{v}^{f} \leq 2 m_{v}, \quad M_{u}^{G, 0} \leq m_{u}-m_{v}, \quad M_{u}^{G, 1}=-\infty, \\
M_{u}^{g, 0} \leq m_{u}, & M_{u}^{g, 1} \leq 0 .
\end{array}
$$

5.3. Parabolic-parabolic systems. We consider the initial boundary value problem corresponding to the following $\mathrm{PDE}$ systems:

$$
\begin{aligned}
& \partial_{t} u+\sum_{|\alpha|=0}^{m_{u}}(-1)^{|\alpha|} \partial_{x}^{\alpha} F_{\alpha}\left(\mathrm{D}_{x}^{m_{u}} u, \mathrm{D}_{x}^{M_{v}^{F}} v, x, t\right)=f\left(\mathrm{D}_{x}^{2 m_{u}-1} u, \mathrm{D}_{x}^{M_{v}^{f}} v, x, t\right) . \\
& \partial_{t} v+\sum_{|\alpha|=0}^{m_{v}}(-1)^{|\alpha|} \partial_{x}^{\alpha} G_{\alpha}\left(\mathrm{D}_{x}^{M_{u}^{G}} u, \mathrm{D}_{x}^{m_{v}} v, x, t\right)=g\left(\mathrm{D}_{x}^{M_{u}^{g}} u, \mathrm{D}_{x}^{2 m_{v}-1} v, x, t\right) .
\end{aligned}
$$

LEMma. The coupling in the above initial boundary value problem is admissible for the following choices of the parameters $M$ :

$$
M_{v}^{F} \leq m_{v}-1, \quad M_{v}^{f} \leq m_{u}+m_{v}-1, \quad M_{u}^{G} \leq m_{u}, \quad M_{u}^{g} \leq m_{u}+m_{v}
$$

or

$$
M_{v}^{F} \leq 2 m_{v}-1, \quad M_{v}^{f} \leq m_{u}+2 m_{v}-1, \quad M_{u}^{G} \leq m_{u}-m_{v}, \quad M_{u}^{g} \leq m_{u}
$$

or

$$
M_{v}^{F} \leq 2 m_{v}, \quad M_{v}^{f} \leq m_{u}+2 m_{v}, \quad M_{u}^{G} \leq m_{u}-m_{v}-1, \quad M_{u}^{g} \leq m_{u}-1
$$

or

$$
M_{v}^{F} \leq 2 m_{v}-m_{u}-1, \quad M_{v}^{f} \leq 2 m_{v}-1, \quad M_{u}^{G} \leq 2 m_{u}-m_{v}, \quad M_{u}^{g} \leq 2 m_{u} .
$$

6. Limitations of our theory. Finally, we want to point out some of the limitations of our theory. Therefore, we make the following definitions:

$$
\boldsymbol{A}(\nabla):=\left(\begin{array}{cc}
\Delta & \Delta \\
-\alpha \Delta^{2} & -\Delta^{2}
\end{array}\right), \quad \boldsymbol{G}(t):=\boldsymbol{F}^{-1} \exp (t \boldsymbol{A}(-i \xi)) \boldsymbol{F} .
$$

In the above definitions $\boldsymbol{A}(-i \xi)$ denotes the symbol of the differential operator $\boldsymbol{A}(\nabla)$, and $\boldsymbol{F}$ denotes the Fourier transform. We consider the following linear initial value problem:

$$
\partial_{t} u=\boldsymbol{A}(\nabla) u,\left.\quad u\right|_{t=0}=v(x) .
$$

In particular, we have:

$$
u(x, t)=\boldsymbol{G}(t) v(x) \quad \forall v \in \boldsymbol{F}^{-1} \mathcal{C}_{0}^{\infty}\left(\mathbb{R}^{n}, \mathbb{R}^{2}\right) .
$$

We note that the above initial value problem has a unique solution in the sense of our general theory if and only if the solution operator $\boldsymbol{G}(t)$ extends to a bounded linear operator on $L^{2}\left(\mathbb{R}^{n}, \mathbb{R}^{2}\right)$. Moreover, we note that in our general theory we look at evolutionary problems from the point of view of perturbation theory. For the case of the 
above initial value problem this means that we consider the diagonal of $\boldsymbol{A}(\nabla)$ as the principal part and the remainder as a perturbation. This approach has the feature, that we obtain existence and uniqueness of solutions either for all $\alpha \in \mathbb{R}$ or only for $\alpha=0$. Now it is easy to see that for some $\alpha_{1} \neq 0$ the solution operator $\boldsymbol{G}(t)$ extends to a bounded linear operator on $L^{2}\left(\mathbb{R}^{n}, \mathbb{R}^{2}\right)$ whereas for some other $\alpha_{2} \neq 0$ the solution operator $\boldsymbol{G}(t)$ becomes unbounded on $L^{2}\left(\mathbb{R}^{n}, \mathbb{R}^{2}\right)$. Consequently, for $\alpha_{1}$ the above initial value problem has a unique solution but nevertheless the approach of our general theory fails.

\section{References}

[1] C. Dafermos, W. Hrusa, Energy methods for quasilinear initial boundary value problems. Applications to elastodynamics, Arch. Rational Mech. Anal. 87 (1985), 267-292.

[2] S. EBenfeld, $L^{2}$-regularity theory of linear strongly elliptic Dirichlet systems of order $2 m$ with minimal regularity in the coefficients, Quart. Appl. Math. 60 (2002), 547-576.

[3] S. EBEnfeld, Nonlinear initial boundary value problems of hyperbolic-parabolic type. A general investigation of admissible couplings between systems of higher order. I. Linear theory, Math. Methods Appl. Sci. 25 (2002), 179-212.

[4] S. EBenfeld, Nonlinear initial boundary value problems of hyperbolic-parabolic type. A general investigation of admissible couplings between systems of higher order. II. Abstract quasilinear theory, Math. Methods Appl. Sci. 25 (2002), 213-240.

[5] S. Ebenfeld, Nonlinear initial boundary value problems of hyperbolic-parabolic type. A general investigation of admissible couplings between systems of higher order. III. Applications, Math. Methods Appl. Sci. 25 (2002), 241-262.

[6] S. JiAnG, R. RACKE, Evolution Equations in Thermoelasticity, Chapman Hall/CRC Monogr. Surv. Pure Appl. Math. 112, Chapman \& Hall, Boca Raton, 2000.

[7] T. Kato, Abstract Evolution Equations and Nonlinear Mixed Problems, Lezioni Fermiane, Accademia Nazionale dei Lincei and Scuola Normale Superiore, Pisa, 1985.

[8] A. MajdA, Compressible Fluid Flow and Systems of Conservation Laws in Several Space Variables, Appl. Math. Sci. 53, Springer, New York, 1984. 